\title{
Filigrane
}

Écoutes psychothérapiques

\section{Oedipe civilisateur menacé}

\section{Maurice Berger}

Volume 19, numéro 1, printemps 2010

Adieu Oedipe, bonjour Narcisse?

URI : https://id.erudit.org/iderudit/044179ar

DOI : https://doi.org/10.7202/044179ar

Aller au sommaire du numéro

\section{Éditeur(s)}

Revue Santé mentale au Québec

\section{ISSN}

1192-1412 (imprimé)

1911-4656 (numérique)

Découvrir la revue

\section{Citer cet article}

Berger, M. (2010). Oedipe civilisateur menacé. Filigrane, 19(1), 49-63.

https://doi.org/10.7202/044179ar

\section{Résumé de l'article}

À partir de ma pratique clinique, je constate que le fonctionnement psychique de certains enfants et adolescents s'est modifié dans notre civilisation, sous l'influence de changements survenus dans la structure familiale, d'une dissolution de nos instances surmoïques collectives, du refus de reconnaître l'inéluctabilité de la perte dans tout destin humain, et sous l'impact de facteurs technologiques tels qu'Internet. Ces changements soumettent le psychisme infantile à une activité de déliaison et/ou l'amènent à privilégier ce mode de fonctionnement par rapport à l'activité de liaison. Dans ces situations, la structure oedipenne, même si elle est toujours présente, ne parvient plus à jouer son rôle organisateur essentiel du psychisme humain car pour se déployer, elle a besoin d'un environnement pare - excitant et porteur de limites cohérentes dans les registres pulsionnels et narcissiques. Diverses " dérives » apparaissent, en particulier les mouvements d'indifférenciation sociétaux éloignent de l'investissement de la conflictualité intrapsychique et tendent vers un appauvrissement de l'imaginaire et une " déliaison " visant à tout réduire à un sexuel brut. Ceci est grave, difficilement réversible, et a des conséquences sur le travail du psychanalyste et sur sa place dans la société.
Ce document est protégé par la loi sur le droit d'auteur. L’utilisation des services d’Érudit (y compris la reproduction) est assujettie à sa politique d'utilisation que vous pouvez consulter en ligne.

https://apropos.erudit.org/fr/usagers/politique-dutilisation/ 


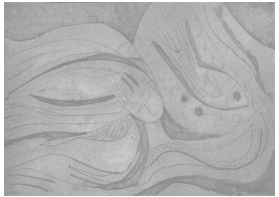

\title{
Edipe civilisateur menacé
}

\section{Maurice Berger}

\begin{abstract}
À partir de ma pratique clinique, je constate que le fonctionnement psychique de certains enfants et adolescents s'est modifié dans notre civilisation, sous I'influence de changements survenus dans la structure familiale, d'une dissolution de nos instances surmoïques collectives, du refus de reconnaître l'inéluctabilité de la perte dans tout destin humain, et sous l'impact de facteurs technologiques tels qu'Internet. Ces changements soumettent le psychisme infantile à une activité de déliaison et/ou l'amènent à privilégier ce mode de fonctionnement par rapport à l'activité de liaison. Dans ces situations, la structure œdipenne, même si elle est toujours présente, ne parvient plus à jouer son rôle organisateur essentiel du psychisme humain car pour se déployer, elle a besoin d'un environnement pare - excitant et porteur de limites cohérentes dans les registres pulsionnels et narcissiques. Diverses "dérives" apparaissent, en particulier les mouvements d'indifférenciation sociétaux éloignent de l'investissement de la conflictualité intrapsychique et tendent vers un appauvrissement de l'imaginaire et une "déliaison" visant à tout réduire à un sexuel brut. Ceci est grave, difficilement réversible, et a des conséquences sur le travail du psychanalyste et sur sa place dans la société.
\end{abstract}

$\mathrm{D}$ ans ma pratique clinique, je constate avec inquiétude la rapidité avec laquelle le fonctionnement psychique de certains enfants et adolescents s'est modifié dans notre civilisation, sous l'influence de changements survenus dans la structure familiale, d'une dissolution de nos instances surmoïques collectives, du refus de reconnaître l'inéluctabilité de la perte dans tout destin humain, et sous l'impact de facteurs technologiques, en particulier d'Internet. Ces changements soumettent le psychisme infantile à une activité de déliaison et/ou l'amènent à privilégier ce mode de fonctionnement par rapport à l'activité de liaison ${ }^{1}$. Il serait trop long d'aborder ici toutes les problématiques qui en découlent. Aussi me contenterai-je d'en évoquer un seul aspect, la grande difficulté à maintenir l'aspect organisateur que joue le complexe d'Edipe. Je partirai pour cela de deux situations banales, au sens où les questions qu'elles soulèvent reviennent avec fréquence dans la pratique quotidienne.

\section{Ludovic $^{2}$}

Madame X a demandé une consultation pour son fils Ludovic, trois ans onze mois, qui présente des troubles persistant depuis la mise en place du mode d'hébergement décrit ci-après. Monsieur Y, père de Ludovic, a exigé l'instauration immédiate d'une résidence alternée paritaire (ou égalitaire) dès que le couple parental s'est 
séparé. Afin d'éviter ce dispositif dont elle pensait qu'il risquait d'être nocif pour son enfant âgé alors de huit mois, Madame $\mathrm{X}$ a accepté que Ludovic aille chez son père un week-end sur deux du vendredi soir au dimanche soir, et la moitié des vacances scolaires par tranches de quinze jours. Dès le début, chaque fois que Ludovic était avec lui, Monsieur Y l'empêche d'avoir le moindre contact avec sa mère. Lorsque Madame X téléphonait, elle tombait toujours sur un répondeur et Monsieur ne rappelait jamais. A la longue, elle a renoncé.

Au début de la consultation, Madame $\mathrm{X}$ m'indique discrètement, pour que son fils ne comprenne pas, que Monsieur Y refusera tout rendez-vous avec moi, car pour lui tout va bien. Je la reçois alors seule, Ludovic attendant dans la salle d'attente avec un autre membre de la famille.

Elle m'explique que Monsieur Y l'a quittée quelques mois après la naissance de leur enfant parce qu'il se sentait délaissé quand elle s'occupait du bébé; il lui aurait dit qu'une femme devait faire passer son enfant au second plan par rapport à son mari. Je n'ai pas la version de Monsieur Y. Ce père aurait exercé ensuite diverses pressions sur son ex-compagne, l'attendant par exemple à la sortie de son lieu de travail, et il est venu plusieurs fois à son domicile, parfois avec son amie, pour crier contre Madame X devant Ludovic. Il ne l'a jamais frappée.

Dès la mise en place du mode d'hébergement décrit ci-dessus, Ludovic a présenté plusieurs symptômes. Il partait chez son père en hurlant et en revenait encore plus mal, l'air perdu, ne parlant pas, ne répondant pas, et il mettait au moins un jour à se réapproprier sa chambre. Il a rapidement présenté des difficultés d'endormissement qui sont toujours aussi intenses actuellement et dont la conséquence est qu'il est fatigué le matin à l'école (il est en classe maternelle et l'enseignante indique qu'il se tient en retrait par rapport aux autres enfants). Chaque soir, il a besoin de ritualiser de manière importante le moment du coucher. Il faut que son doudou soit toujours exactement dans la même position. Il répète de nombreuses fois la question «Rien ne va venir?», et demande que la porte de sa chambre soit poussée mais pas fermée, nous comprendrons pourquoi. Peu avant de partir chez son père, il dit à sa mère avoir peur qu'elle ne monte au ciel pendant son absence, et par conséquence, qu'il ne l'a retrouve pas quand il reviendra chez elle. Et dès qu'il a su parler, il a demandé à sa mère pourquoi elle le laissait partir. Madame X espérait que ces troubles s'estomperaient avec le temps, ce qui n'a pas été le cas. Malgré le mal-être de son enfant, elle n'a pas sollicité de modification du mode d'hébergement, craignant que Monsieur Y ne se mette en colère et ne redemande une résidence alternée, ce qu'il entend faire dès l'entrée de son fils à l'école primaire à six ans. Elle précise n'avoir jamais critiqué son ex-compagnon devant Ludovic afin de ne pas mettre son fils dans une situation difficile. Lorsqu'il lui a reproché de ne pas lui téléphoner pendant son absence, elle ne lui a pas expliqué avoir tenté de le faire pour ne pas avoir l'air de dire du mal de Monsieur Y.

Ludovic n'a pas présenté d'asthme, d'eczéma, sa mère ne le trouve pas déprimé, il est surtout angoissé et il pose beaucoup de questions sur la mort.

Ce garçon est ensuite reçu seul. Je constate rapidement qu'il présente une hypermaturité, comme c'est fréquemment le cas chez les enfants qui ont dû affronter trop 
tôt certaines épreuves dans leur existence et qui y ont réagi par un effort intellectuel énorme pour tenter de donner un sens à ce qu'ils ne peuvent que subir. Quand je lui demande pourquoi il pense que ses parents se sont séparés, il répond «C'est parce que papa grondait maman », et il se rappelle bien les moments de violences verbales de son père. Il explique qu'il a toujours pu apporter son doudou et sa sucette chez son père, mais que ce dernier les lui enlevait à son arrivée et les remplaçait par des objets strictement identiques. Il commente: "Il y a le doudou de papa et le doudou de maman. » Je lui fais remarquer que d'habitude si un enfant tient autant à son doudou, c'est parce que c'est le sien et pas celui de papa ou de maman, et qu'il peut le garder avec lui partout. Il acquiesce. Madame X. confirmera cette alternance des doudous et ajoutera que malgré cela, elle fait attention de mettre dans les affaires de son fils au moment du changement d'hébergement, un petit objet qui fasse le lien entre les deux domiciles, une petite voiture par exemple. Je l'interroge: «A-t-il demandé à son père pourquoi il devait changer de doudou et pourquoi il ne pouvait pas parler à sa mère au téléphone?» Il répond qu'il a oublié de le demander.

Quand je le questionne sur ce qu'il pense de la manière dont il vit, il répond qu'il est trop petit pour changer d'endroit comme cela et qu'il a peur. Il ajoute craindre que sa mère ne meure et que ce ne soit la compagne actuelle de son père qui s'occupe de lui, car il ne l'aime pas beaucoup. Il a de la difficulté à se rappeler de quoi il a peur le soir lorsqu'il est chez son père, et indique que Monsieur Y. refuse de mettre une veilleuse dans sa chambre malgré ses inquiétudes. Par contre il se rappelle de quoi il a peur le soir lorsqu'il est chez sa mère : il craint que ne rentrent dans sa chambre un tigre ou un loup qui pourraient le mordre. Et il ne veut pas que sa porte soit totalement fermée afin que Madame X puisse l'entendre s'il appelle au secours.

Je vais chercher Madame X dans la salle d'attente et reprend devant Ludovic ce qu'elle a dit précédemment, en particulier qu'elle a téléphoné mais qu'elle tombait sur le répondeur. Ainsi pendant son absence, elle pensait à lui, il lui manquait, et elle était mal, inquiète car elle savait qu'elle lui manquait. Ludovic écoute attentivement. Quand je lui demande alors s'il souhaite poser des questions, il prononce une phrase stupéfiante: "Comment maman a-t-elle fait pour revenir?» Comme je ne comprends pas, il précise: «Comment maman a-t-elle fait pour revenir de la salle d'attente au bureau (de consultation)?» La question ne se situe pas à un niveau rationnel: les pièces ne sont distantes que de quelques mètres, les enfants petits repèrent aisément l'itinéraire, Ludovic l'a déjà fait deux fois au cours de la consultation, il vient de me voir aller chercher sa mère et a donc pu penser qu'elle m'a suivi pour revenir dans mon bureau.

Je lui demande alors s'il pensait que sa mère n'arriverait pas à le retrouver, et il répond que oui. Je lui demande ensuite si, de la même manière, lorsqu'il était chez son père, il pensait qu'elle l'avait perdu et que lui l'avait perdue. Il répond là aussi positivement. J'évoque alors la manière dont il craint que sa mère ne meure. S’il a en permanence cette peur, c'est parce que dans son esprit, il a déjà perdu complètement sa mère de nombreuses fois. Sur une feuille, je dessine une succession de semaines et lui explique qu'un enfant de huit mois et même plus âgé ne peut garder l'image de 
sa maman dans la tête que pendant un certain nombre de jours; au-delà, cette image s'efface et c'est comme si sa mère n'existait plus pour lui. C'est sans doute ce qui s'est passé pour lui, en particulier lorsqu'il restait quinze jours sans voir ni entendre sa mère. Il perdait aussi l'image de sa maison puisqu'il ne reconnaissait pas sa chambre lorsqu'il revenait. Ce n'est que lorsqu'on est plus grand que l'image reste imprimée sans s'effacer, comme la photo d'un visage que je lui montre sur une revue. Et il y a toujours en lui un Ludovic petit qui garde ses peurs du passé.

De plus, il imaginait que la même chose se passait pour sa mère, c'est-à-dire qu'elle perdait l'image de Ludovic puisqu'il pensait qu'elle ne lui téléphonait pas. Et c'est pourquoi il croyait qu'elle ne pourrait pas retrouver le bureau où il est et revenir le chercher. Je demande à Madame X ce qu'elle a envie de dire, et elle répond qu'au contraire, elle pensait sans cesse à son enfant dont l'image ne s'effaçait pas dans son esprit.

Je laisse de côté plusieurs autres aspects de cette consultation (que représente pour lui le fait de voir un homme et une femme réfléchir tranquillement ensemble sur ce qu'il ressent?) pour souligner simplement que la vie psychique de cet enfant est cadenassée par un interdit de penser. Chaque fois que j'ai évoqué les questions qu'il pourrait poser à son père, sa réponse a été "J'ai oublié». À la fin de la consultation, Madame X me demande de ne pas revoir Ludovic car elle a peur des représailles de Monsieur Y, opposé à ce genre de rendez-vous. Je téléphone quinze jours plus tard à cette mère pour savoir si la consultation a pu soulager les symptômes de son fils, et sa réponse est négative.

Cet exemple souligne les effets possibles de l'indifférenciation père-mère comme nouveau mode de «coparentalité ».

\section{Julie}

Julie, âgée de dix ans et demi, incitée par une camarade à surfer sur Internet en donnant un faux âge pour avoir accès à un site apparemment anodin, entre ainsi en contact avec un garçon inconnu de 17 ans qui lui propose d'établir une liaison par webcam. Au bout de quelque temps de conversation, elle accepte de «jouer » à se mettre partiellement torse nu devant lui pendant quelques secondes en soulevant le devant son tee-shirt. Elle est pré pubère et n'a pas de poitrine. Le garçon sort alors son sexe et se masturbe devant la webcam. Paniquée, Julie ferme l'ordinateur. Elle rompt la relation avec sa camarade, mais elle est en état de choc pendant les deux mois qui suivent, ce qui motive la consultation. Elle pleure beaucoup, a perdu l'appétit, ne trouve pas le sommeil. Elle se sent coupable de ne pas avoir arrêté l'échange plus tôt, les images tournent dans sa tête et elle ne voit pas comment s'en sortir. Elle se dit que si elle se tue, elle n'y pensera plus, mais qu'elle n'a pas envie de mourir. De plus, elle se pose beaucoup de questions par rapport à sa sexualité et pense à ses jeux d'enfant avec un autre regard: "Suis-je homo, car quand j'étais petite, on jouait à papa et maman avec une copine et on se montait l'une sur l'autre?»; "J'ai peur de tomber amoureuse d'un cousin », comme si elle était tout à coup «hypersexuée ». Ce qui était au départ un jeu de séduction transgressif devient un traumatisme psychique lorsque surgit la confrontation à un acte sexuel réel, ce qui amène à une 
culpabilité œedipienne: elle est punie d'avoir voulu faire comme les grands (en trichant sur son âge), sauf que ces derniers ne montrent pas leur poitrine à un inconnu. Le dispositif «technologique» a court-circuité le temps d'appropriation progressif des fantasmes et des conduites de séduction de bon aloi, privant ainsi Julie de l'étayage de sa mère qui, quelques années plus tard, aurait pu l'aider à choisir les habits et le maquillage qui lui vont bien au lieu de se dévêtir devant un inconnu, ce qu'elle n'aurait pas fait si elle avait été en contact direct avec ce garçon. Au contraire, la fille et la mère ont le sentiment de s'être perdues l'une l'autre. En effet, la mère est également traumatisée. Elle trouve que Julie n'est plus la petite fille qu'elle connaissait, elle a "carrément changé, n'est plus enthousiaste». Madame a pleuré plusieurs fois suite à cette histoire dont elles reparlent beaucoup toutes les deux.

\section{Le complexe d'Fipe: un organisateur essentiel du psychisme humain}

Pour aller plus loin, il faut rappeler brièvement certaines caractéristiques du complexe d'Edipe.

Du côté de l'enfant, il s'agit, entre autres, de l'apparition de quatre désirs. 1) Désir de faire couple avec le parent du sexe opposé. 2) Désir de faire couple avec le parent du même sexe. À noter que dans ces deux mouvements affectifs, un parent est intéressant à désirer parce qu'il est le mari ou l'épouse de l'autre. 3) Désir d'être au centre du couple parental qui l'a créé. 4) Désir de détruire ce couple qui risque de l'exclure. Contentons-nous de réfléchir au premier désir, lui-même à l'origine d'un sentiment de culpabilité et de l'angoisse de perdre l'amour de l'autre parent ou des parents ou de subir une rétorsion. Quels compromis vont permettre à l'enfant de trouver une issue? Principalement quatre déplacements :

- Déplacement du désir sur un autre objet : «Ce n'est pas avec mon père (ou ma mère) que je ferai un couple, mais avec une autre personne »;

- Déplacement dans le temps : «Ce n’est pas à mon âge que je peux constituer un couple mais plus tard, quand je serai adulte», raisonnement qui implique la reconnaissance de la différence des générations.

- Déplacement de l'acte à la pensée: «Je ne peux pas réaliser en vrai mes désirs sexuels ou meurtriers, mais je peux les penser, les fantasmer de diverses manières ", ce qui s'accompagne d'un investissement accru de l'activité de représentation;

- Déplacement de la pulsion vers un but non sexuel, c'est la sublimation.

Ces déplacements sont favorisés par le fait d'avoir des parents «œedipiens» au sens structurel et non pulsionnel du terme. Il s'agit de parents qui vivent en couple, ou s'ils sont séparés, qui ne dénigrent pas le couple qu'ils ont formé, qui tiennent un rôle de pare-excitation, permettant ainsi que s'installe la phase de latence, et qui assument une position surmoïque à la fois ferme et non tyrannique. Voyons ces éléments en détail.

Tout d'abord, l'enfant a besoin de constater que le couple résiste à ses attaques et à ses désirs, et que ses pensées ne détruisent pas le lien entre son père et sa mère. 
La structure œedipienne n'existe donc pas seulement comme fantasme dans l'esprit de l'enfant, mais aussi comme cadre (Donnet, 1993). Ce cadre est nécessaire pour que l'enfant puisse réaliser un travail psychique difficile: tenir ensemble la représentation d'un couple de parents et la représentation d'un couple sexué hommefemme, en faisant en sorte que ces deux fonctions, parentales et sexuelles, soient complémentaires et ne disjonctent pas. L’enfant sait qu'il a été conçu et accueilli par ses parents. S'il se sent bien avec eux, c'est parce qu'il peut fantasmer une scène primitive trouvée-créée, transitionnelle, indique Roussillon (1988) : lorsque ses parents l'ont conçu au cours d'une relation sexuelle, il était avec eux sans y être. Ils ont en même temps envie d'être parents, d'élever leur enfant à deux, et de préserver un espace pour leur couple. Ils excluent donc à certains moments l'enfant de leurs activités ou discussions, de manière non excessive, tout en gardant une préoccupation parentale pour lui. Et, ajoute cet auteur, c'est parce qu'il est ainsi seul en présence/absence du couple que l'enfant peut se questionner sur le plaisir que ses parents prennent ensemble et se constituer ce qu'on nomme des fantasmes originaires qui portent sur la manière dont les enfants sont conçus, dont les hommes et les femmes se séduisent, et sur la différence des sexes.

On peut dire que pour l'enfant, ses parents sont des amoureux pas comme les autres. Il peut ricaner en regardant des amoureux s'embrasser sur un banc public, tout en refoulant le fait que son père et sa mère font la même chose le soir dans leur chambre. Pour un enfant, la structure oedipienne de la famille est ce qui existe de mieux pour tenir ensemble le besoin de sécurité, la tendresse, et le sexuel. La recomposition familiale, devenue fréquente, va dans le sens inverse, en créant, dans «la chambre à côté », un couple qui n'est que sexuel aux yeux de l'enfant, ce qui peut lui être difficilement supportable (il s'agit là d'une constatation et non pas d'une injonction de ma part à ne pas refaire sa vie après un divorce).

Cela ne signifie pas non plus qu'un enfant de parents divorcés ne pourra pas construire une problématique œdipienne ni y trouver une issue, mais le divorce ne lui facilite pas la tâche, en le mettant en position de «couple» lorsqu'il est seul avec un parent, les moments de câlins pouvant prendre une tonalité inquiétante dans l'esprit de chacun en l'absence d'un tiers. Ou parce que l'enfant est mis face au choix suivant : être avec un parent, c'est faire disparaître l'autre de son esprit. Ainsi on ne voit pas comment Ludovic va pouvoir construire un complexe d'Edipe cohérent puisque son père lui interdit d'évoquer sa mère et d'y penser. Et même lorsqu'il n'est pas là, ce père surgit le soir dans son esprit sous la forme d'un tigre ou d'un loup, père qui ne veut rien savoir de l'autre membre du couple originaire. Et concernant sa mère, il est possible que Ludovic, au lieu d'éprouver des sentiments amoureux, soit d'abord préoccupé par la crainte qu'elle ne tombe malade en constatant que son enfant va mal; ceci n'est pas rare chez les mères qui ne supportent pas de voir leur nourrisson être en proie à des troubles psychiques et psychosomatiques importants suite à la mise en place d'une résidence alternée.

Même lorsque les parents divorcés ne sont pas en conflit concernant la garde de leur enfant, la situation est compliquée à un niveau intrapsychique. Écoutons Marie, 
élevée en résidence alternée paritaire consensuelle à partir de l'âge de huit ans. "J'ai le sentiment d'avoir été sans arrêt déracinée, sans arrêt baladée d'un point à un autre, de n'être nulle part chez moi. Je ressentais bien que j'aurais préféré «nicher» chez ma mère, non pas que j'éprouvais moins d'amour pour mon père, mais je ne me l'explique pas, ma base de sécurité affective était naturellement là. Ce n'est certainement pas parce que j'aurais préféré avoir une maison fixe que j'en aurais moins aimé mon père. Chaque séparation d'avec ma mère était douloureuse et vécue comme une déchirure, j'étais arrachée ou peut-être je devais m'arracher à sa tendresse, à ce qui me rassurait, avec toujours la crainte de la perdre, et la peur panique que quelque chose n'arrive et qu'elle ne puisse pas revenir. Je n'avais jamais l'esprit libre. Je vivais en permanence avec un fond d'angoisse d'abandon. J'ai développé des sortes de tocs, je me répétais sans discontinuer au cours de la journée des petites phrases rituelles qui me rassuraient, je prenais systématiquement le même chemin pour rentrer de l'école, sans même changer de trottoir par crainte... d'un mauvais sort. Je me rappelle également que chaque retrouvaille avec ma mère était douloureuse, difficile et conflictuelle: je lui en voulais et lui faisais payer le fait de m'avoir laissée, je n'arrivais pas à lui exprimer autrement mon chagrin. Malgré une psychothérapie, je garde en moi, à trente-cinq ans, un fond d'angoisse permanent, toutes mes bases ont été touchées. Je commence seulement à découvrir ce que signifie le mot «se détendre «. On mesure quel cheminement Marie devra encore effectuer pour accéder à la capacité de se construire «un projet de vie œdipien ", au lieu de lutter en permanence contre le sentiment de perte de l'objet. Il ne s'agit pas d'un problème mineur: en France les couples se séparent de plus en plus tôt après la naissance d'un enfant, $4,2 \%$ de nourrissons âgés d'un an, et $11 \%$ des enfants de six ans vivent en résidence alternée paritaire, et même lorsque ce mode de garde est mis en place de manière non conflictuelle pour des enfants âgés de plus de trois ans, des travaux récents montrent que le vécu de perte répétée des lieux et des personnes entraîne une souffrance importante. L'enfant, préoccupé de ne pas décevoir ses parents et pris dans un «syndrome de l'enfant parfait», ne révèle pas à ses père et mère ses difficultés, en particulier sa dépression, auxquelles seul un psychothérapeute peut avoir accès (Levy-Soussan, 2006, Izard, 2009).

Deuxième élément, le parent « œdipien » est pare-excitant en respectant la mise en latence des pensées sexuelles de son enfant: pour cela, il reste sourd à la dimension sexuelle des demandes de l'enfant et refoule ses propres pensées sexuelles en sa présence (Aulagnier, 1984). Cette période de latence représente une accalmie pulsionnelle, même si elle n'est que relative, caractérisée par une valeur nouvelle donnée à l'activité de représentation (Roussillon, 2007). Or la société, devenue séductrice, accepte actuellement que l'enfant soit constamment soumis à des excitations de nature sexualisée, par le biais d'images publicitaires, de bandes annonces de films, et par Internet. Un jeune sur deux a déjà vu un film pornographique à l'âge de dix ans (Levasseur, 2009) et de nombreux auteurs soulignent qu'il s'agit d'une véritable effraction, de l'intrusion d'une problématique d'adultes dans le monde des enfants avec un effet littéralement traumatique qui se traduit parfois par des syndromes de stress, ou par un désinvestissement scolaire, un refuge dans l'imaginaire, etc., et par 
une représentation de la sexualité des parents très crue, n'ayant aucun rapport avec ce que l'enfant avait imaginé. Ainsi une fille de dix ans demande à sa mère, stupéfaite, si «papa lui lèche la chatte». L'exemple de Julie a montré les effets de la prématurité de l'apparition des images sexualisées. La situation de Jacques la met aussi en évidence d'une manière différente.

Jacques, sept ans, admis quotidiennement en hôpital de jour pour une dysharmonie psychotique avec des angoisses à la limite de l'hallucination et un comportement à la fois violent et très régressif, parvient à organiser sa pensée au bout de deux ans de soins. Simultanément, les entretiens familiaux permettent au père de prendre une position paternelle étayante et ferme alors qu'auparavant, il permettait tout à son enfant, sabotant les tentatives d'autorité de son épouse et nous regardant avec incrédulité tenter de démêler l'indifférenciation régnant entre les psychismes de chacun des membres de la famille. L'« œedipification » de la pensée de Jacques apparaît dans les scénarios qu'il apporte au cours des entretiens successifs. 1) Tout d'abord un voleur nargue un père de manière répétitive. 2) Puis un enfant, Kévin, grandit, devient adolescent, a 17 ans et veut faire le métier d'éducateur (comme son éducatrice). La femme qui est sa professeure est l'épouse du maître que Kévin avait plus petit (représentation d'un couple sympathique qui l'aide à grandir, au moment où ses parents réussissent à former enfin un couple éducatif). 3) Il décide de quitter le domicile de ses parents et de vivre dans un appartement à lui. 4) Jacques déclare à son éducatrice: "Il lui faut une femme, à Kévin. Tu sais comment il faut faire?» L'éducatrice lui demande ce qu'il en pense. Il réfléchit, dit «Il faut qu'ils s'aiment», et... au lieu qu'apparaissent des fantasmes originaires, il ajoute « comme à la télé où j'ai vu la sexy dance, un homme qui lèche les seins de la dame où il y a avait de la crème chantilly». Surprise, l'éducatrice demande des explications et apprend que la sœur de Jacques, âgée de quatorze ans, regarde à la télévision à 18 heures, heure de grande écoute, une émission pour adolescents intitulée : «Sexy danse » dans laquelle des jeunes, hétéro ou homosexuels, font des « expériences ». Ainsi un garçon a mis de la crème chantilly sur la poitrine d'une fille en soutien-gorge et l'a léchée. L'éducatrice téléphone alors aux parents de Jacques en leur suggérant de prêter attention aux émissions que leur fille fait regarder à son frère, tout en leur demandant que Jacques ne soit pas au courant de cette conversation téléphonique qui fait référence à des paroles qu'il a prononcées dans l'intimité de la prise en charge. C'est ce qu'ils feront. Mais si Jacques, avec une certaine innocence, est surtout sensible à la dimension régressive de la scène, la chantilly, on constate comment son mouvement élaboratif bute transitoirement sur l'excitation brute transmise par les médias et autorisée socialement, et sur un modèle de satisfaction immédiate des désirs, contrairement à l'investissement qu'il est en train de faire de l'activité de pensée, dans un mouvement de mise en latence. Avec l'aide de l'attitude pare-excitante de ses parents et de l'éducatrice, différente de l'effondrement de la mère de Julie qui a vécu l'irruption du sexuel comme une différenciation brutale, Jacques crée une autre solution: Kévin entre dans une école de formation d'éducateur et, en discutant, trouve une compagne gentille, jolie, et vêtue d'un pull rose. 
Enfin le complexe d'CEdipe ne peut trouver de solution que si l'enfant rencontre un Surmoi parental suffisamment solide, émanant d'un parent qui assume l'asymétrie entre parents et enfant. Or dans la société française, le Surmoi s'est « dissout ». Les interdits sont souvent disqualifiés en étant dénommés « retour à l'ordre moral ». On constate alors que beaucoup de parents n'ont pas intériorisé une fonction surmoïque cohérente et sont incapables de tenir un interdit. Il y a une trentaine d'années, ils parvenaient à exprimer leur autorité, mais c'était grâce à l'étayage groupal extérieur, en s'appuyant sur la crainte du regard d'autrui, du qu'en dira-t-on, ce dont on ne se rend compte que depuis que la transmission intergénérationnelle du Surmoi s'est affaiblie. Le résultat en est la perte d'une censure de bonne qualité qui permet de différencier l'interdit de faire, l'interdit de dire publiquement, actuellement confondus avec un interdit de penser qui lui, serait tyrannique; une confusion entre «autorité» et "abus d'autorité» avec une difficulté majeure à penser la valeur des limites; la perte du sentiment de dette non aliénante et de gratitude («s'il te plaît», «merci»), la dépendance liée à la demande étant remplacée par l'exigence; et la perte du sentiment de transgression. Je me rappelle avoir vu mes premières images osées à l'âge de 15 ans. C'était dans une revue qui paraîtrait bien innocente maintenant, et que m’avait prêtée un cousin plus âgé. J'étais très embêté d'avoir ce journal entre les mains. Je savais que c'était interdit, et cette idée était essentielle. L'important n'était pas tellement d'avoir des fantasmes sur le sexe, mais le fait que je faisais cela en cachette. Actuellement, le sentiment de honte structurante, différente de l'humiliation, s'estompe. Au contraire, certains jeunes et parents auraient honte d'avoir honte.

\section{La dissolution de la structure œdipienne}

L'absence fréquente de positionnement paternel avec une non-différenciation des rôles contribue à l'augmentation des phobies scolaires, alors qu'une des fonctions du père est de soutenir l'enfant dans son exploration du monde extérieur, hors de la douceur du monde maternel. Ainsi après quinze jours d'hospitalisation dans notre service de pédopsychiatrie, un garçon de dix ans qui présente une telle phobie est autorisé à aller le week-end dans sa famille. Il refuse de revenir à l'hôpital le lundi et au bout de quelques jours, nous demandons au juge des enfants d'envoyer la police le chercher à son domicile pour qu'il soit hospitalisé sous contrainte. Dans cette situation particulière, la phobie cessera "par peur du gendarme». Mais plus encore, l'absence de positionnement paternel peut entraîner un CEdipe exacerbé. Un autre enfant de onze ans qui présente lui aussi une phobie scolaire est amené dans le service par sa mère pour être hospitalisé, son père ne prenant pas position dans cette décision. Au cours de l'entretien familial suivant, le garçon, très en colère, déclare à sa mère qu'elle l'a trahi et exige des excuses de sa part, qu'il n'obtient pas. Il décrète alors une grève de la faim, devant le père qui reste silencieux.

De plus en plus de parents, père ou mère, excusent les mouvements pulsionnels de leur enfant en évoquant une mentalité de groupe (Bion, 1959; Guignard, 2009). Ceci montre que le maintien d'une cohérence psychique repose sur peu d'individus dans une société, et que pour les autres, contenir ses pulsions n'est qu'un vernis qui 
dépend du support collectif. On peut évoquer ici la désillusion causée à Freud par la conduite des Etats et des hommes lors de la première guerre mondiale (1915) : «Au sein de chacune des grandes nations avait été établies, pour l'individu, des normes morales élevées, auxquelles il devait se conformer dans la conduite de sa vie, s’il voulait trouver sa place dans la communauté civilisée [...]. Les motions pulsionnelles ne sont en soi ni bonnes ni mauvaises. Nous les classons comme telles en fonction de leur rapport avec les besoins et les exigences de la communauté humaine. »

Je ne dirai pas pour autant que tout élément œedipien a disparu du fonctionnement psychique d'un certain nombre d'enfants et d'adolescents que je reçois en consultation. Un tel matériel apparaît en permanence au cours des psychothérapies et autres modes de prises en charge thérapeutiques sous la forme de jalousie, d'un désir d'éliminer ou de ridiculiser le rival. La question qui se pose est de parvenir à aider ces sujets à organiser ces spores d'Edipe sous la forme d'un complexe qui devra tenir et contenir les éléments décrits précédemment.

Lorsque les contraintes liées à la nécessité de trouver une issue au complexe d'Edipe ne sont plus les organisateurs du psychisme, apparaît le risque d'un fonctionnement narcissique marqué par un fantasme d'auto-engendrement (s'être fait tout seul en utilisant le corps de l'autre, spermatozoïde, ovule, ou utérus, faire son enfant tout seul, ne rien devoir à qui que ce soit); et par un fantasme d'immortalité qui amène à nier toute perte synonyme d'incomplétude et de finitude, donc à la limite toute pensée sur l'objet qui se prétendrait distinct de soi. Lorsque sa compagne s'occupe de leur bébé, le père de Ludovic ne le supporte pas car il n'est plus au centre des regards, et il ne peut pas être heureux de constater que sa concubine est une mère attentionnée pour leur enfant. Il fait disparaître la mère de sa pensée et veut qu'elle disparaisse aussi de celle de son fils. Mais il y a plus. Au cours d'une expertise, en s'appuyant sur la loi actuelle, un père demande une résidence alternée pour son enfant âgé d'un an. Il m'explique qu'il voulait avoir un enfant, un point c'est tout, et peu importait que ce soit avec cette femme-là ou avec une autre. Mais la femme, utilisée comme une sorte de mère porteuse, ne le savait pas: elle désirait un enfant avec cet homme-là, et ne réalisera à quoi elle a été utilisée qu'au moment de la naissance ou peu après. Du fait de leur histoire personnelle, de tels hommes, qui font un enfant tout en niant la différence des sexes, considèrent qu'un père peut suffire et ils préfèreraient être seuls à élever l'enfant, mais ils savent que cela ne sera pas accepté socialement et judiciairement. Leur demande de résidence alternée est pour eux un moindre mal.

L'étape supplémentaire concerne l'utilisation faite des méthodes de procréation médicalement assistée par certaines personnes, heureusement pas par toutes celles qui y ont recours: la technique leur permet de nier le fait qu'on ne peut faire un enfant tout seul. Comme l'indique P. Levy Soussan (2008), «les progrès techniques en matières de procréation médicalement assistée ont permis de passer du «sex without children » avec la contraception au « children without sex «», ce qui a comme résultat d' " atomiser les éléments liés de façon intime et privée dans les filiations classiques, filiation, désir, intimité, homme, femme, couple, acte sexuel, fécondation, 
gamètes, embryon, enfant». Un exemple des conséquences cliniques qui en découlent sera donné plus loin.

Il faut ajouter un autre élément souvent lié à la prédominance d'un fonctionnement narcissique : la dimension perverse, facilitée et attirée par la place de l'image, objet d'élection de la pulsion scopique, pulsion en place du regard introspectif. Citons simplement ici la figure de l'initiateur (trice). Pour Julie, il s'est agi de sa camarade et du garçon de 17 ans; pour Jacques, c'était sa sœur; pour moi, un cousin. L'initiateur (trice) éprouve une jouissance à traumatiser l'autre en le soumettant à un excès d'excitation. Il s'agit d'une sorte d'exhibitionnisme sexuel destiné à choquer l'autre par l'intermédiaire d'un écran ou d'un journal.

\section{La place du psychanalyste}

Quelle est la place du psychanalyste face à cet ensemble de changements? C'est à lui que famille et enfant vont demander plus ou moins consciemment de tenir le rôle de contrepoids face à la mentalité de groupe.

1) En ce qui concerne le travail psychothérapique individuel avec l'enfant, F. Guignard souligne les difficultés auxquelles le praticien va se heurter chez ces jeunes patients : épistémophilie remplacée par le sadisme primaire à l'œuvre dans les jeux vidéo et sur Internet, investissement de l'action et recherche de stimulation au détriment de l'investissement des émotions et du monde psychique interne, développement du monde de l'image imposée à la perception visuelle au détriment du développement du langage intérieur. Ainsi au cours d'un entretien, alors que je l'incite à penser, à «imaginer », un adolescent me répond: «Les idées, ça ne sert à rien, ça n'a pas grande importance, ça ne va pas m'apporter grand chose dans la vie»; pour lui, l'image a remplacé l'imaginaire. Je lui réponds: «Sans possibilité de jouer avec des idées dans son esprit, on est tout le temps obligé de chercher des objets ou des personnes à l'extérieur de soi pour éprouver du plaisir, ou de se procurer des excitations de diverse manière, jeux vidéo, etc., pour se sentir vivre. Donc finalement, on dépend beaucoup du monde extérieur alors qu'on pense être indépendant». L'enjeu, c'est évidemment la possibilité de s'être constitué des auto-érotismes par reprise du plaisir éprouvé à l'origine avec les objets primaires. Pour pouvoir jouer avec ses pensées, il faut avoir acquis, dès les premiers mois de la vie, lors de jeux créatifs et non excitants avec ses parents, la capacité de faire semblant, ce qui est différent de «faire pareil que» ou de confondre image et réalité. Il est probable que Ludovic, qui lutte en permanence contre le sentiment de perte depuis l'âge de huit mois, n'a pu que partiellement se constituer de tels érotismes. Dans ces contextes cliniques, F. Guignard décrit l'intervention du psychanalyste comme tentant de proposer un «rattrapage spéculaire», mais cet auteur se demande en même temps si l'organisation d'une vie psychique interne sera toujours intéressante aux yeux des générations à venir?

2) Au niveau du travail thérapeutique avec le groupe familial, j'indiquais en 1986 ma réticence, en tant que psychanalyste, à proposer des conseils de type structuraux destinés à établir une limite entre les générations et se situant donc sur un versant 
assez éducatif. En effet, ces conseils se révélaient souvent inefficaces et ne constituaient qu'une prothèse externe non intériorisée. J'affirmais même que les conseils étaient la faillite de la pensée et préférais faire appel au «tiers interne» présent dans le fonctionnement psychique du parent, en évoquant «l'enfant dans le parent », ce qui lui a manqué, ce qu'il a subi, ce qui l'a séduit, de manière à permettre au parent de s'identifier à son enfant réel et à ses besoins (de limites, de protection, etc.). Lorsqu'on utilise l'outil analytique, la question n'est pas tant «que faire? » mais « qu'est-ce qui empêche de faire, d'agir ce qui est déjà présent en soi? «Ceci était facilité par le fait que les parents ressentaient un décalage entre leur mode éducatif ou le comportement de leur enfant, et les normes sociales. Depuis quelques années, j’ai été obligé de changer de point de vue dans un certain nombre de situations où dans l'esprit des parents, la mentalité de groupe pré-œdipienne l'emporte sur l'évocation du tiers interne. Dans ces cas, l'enfant s'est lui-même organisé solidement sur ses « avantages sociaux acquis » et n'est pas prêt à perdre quoi que ce soit de sa toute-puissance, futce au détriment de ses capacités de création et d'autonomie. Il peut alors être nécessaire de proposer un contrat écrit élaboré avec les parents, sorte de prescription «médicale ( qui n’a rien à voir avec les prescriptions paradoxales systémiques) destinée à donner aux parents le point d'ancrage dont ils avaient une sorte de préconscience mais qui ne rencontrait pas d'écho dans leur environnement proche ou social. Les parents s'appuieront sur ce contrat à la maison en évoquant ce qui a été décidé au cours de la consultation précédente et ce qui en sera dit lors du rendezvous suivant. Ils se trouvent alors dans une situation équivalente à celle de certaines mères qui déclarent à leur enfant «J'en parlerai à ton père quand il rentrera du travail, tu verras ce qu'il en pensera ». Ce n'est que dans un second temps, lorsque les agirs de l'enfant sont plus contenus, que le travail élaboratif peut se mettre en place.

3) Le troisième point concerne la place du psychanalyste face à «l'opinion publique» que Freud (1914) décrit comme une des instances de censure. Il faut souligner ici le rôle négatif de certains médias qui se caractérise par une dérision gratuite. Cette perversion de l'humour est une forme de déliaison qui consiste à déformer par l'ironie toutes les tentatives de différenciation et de pensée sur les limites, activités de liaison toujours imparfaites et à recommencer indéfiniment. La dérision et l'intention destructrice qui l'accompagnent sont les modes de fonctionnement psychique les plus immédiatement disponibles au psychisme humain et les plus économiques en énergie à court terme. Mieux vaut détruire ce qui est imparfait et laborieux chez l'autre, et garder ainsi l'illusion de solutions idéales obtenues sans effort. A l'opposé, Freud (1920) termine "Au-delà du principe de plaisir» par la citation d'un poète, Rückert: «Ce qu'on ne peut atteindre en volant, il faut l'atteindre en boitant. Boiter, dit l'Ecriture, n'est pas un péché. » Il est frappant de constater comment ce qui exacerbe le plus la destructivité chez autrui, ses attaques envieuses, c'est la cohérence de la pensée chez un sujet concernant l'inéluctabilité de se confronter à la limite, limite de ce qui est permis ou non ${ }^{3}$, limite de la capacité d'accueil sur un territoire, impossibilité de garder dans la réalité tous les liens avec les personnes rencontrées depuis son enfance, donc aspect inévitable du chagrin. P. Aulagnier (1984) y ajoute les trois 
traumatismes inhérents à la condition humaine: l'altérité, la différence des sexes, l'inévitabilité de la mort.

4) Ne serait-il pas souhaitable d'indiquer publiquement que les psychanalystes et psychothérapeutes ne peuvent pas garantir qu'ils seront à même de traiter toutes les difficultés psychiques liées à certaines technologies procréatives ou à certaines pertes des repères sexuels. Ainsi une femme me demande par écrit un rendez-vous pour des entretiens familiaux: "Je suis nouvelle mère d'une petite fille, Claude, âgée de huit mois que j'élève avec ma compagne. Cette enfant a été créée par insémination artisanale ( sic) suite au don de sperme d'un «collègue ami». Depuis sa naissance, il s'avère que le géniteur nous impose un avocat pour pouvoir "récupérer» l'enfant chez lui trois jours tous les quinze jours, en voulant imposer ses droits et responsabilités que la loi lui confère. Un contrat initial où il s'engageait à n'avoir ni droit ni responsabilité a été rédigé à l'origine de notre projet. Mais voilà, j'ai découvert que cet homme mentait. Ma compagne, parente sociale, et moi sommes contraintes de faire intervenir un juge des affaires familiales. Notre demande est impérativement une médiation familiale, une expertise psychologique, et nous souhaiterions entreprendre un travail de thérapie familiale avec vous pour démêler les psychés des membres de la famille. A travers toutes ces démarches, nous espérons pouvoir mettre en place un projet qui permettrait à Claude d'évoluer positivement et non pas de subir plus que de mesure les nœuds personnels et interpersonnels existants».

La surcharge de mon emploi du temps m'a empêché de répondre positivement à cette demande, mais on peut remarquer les questions qu'elle soulève. Le travail de liaison/différenciation qui est sollicité ici est-il réalisable? Quel ensemble d'individus appelle-t-on famille? Comment cet enfant que chacun a fabriqué pour soi en utilisant le corps d'un autre pourra-t-il se représenter une origine, un désir fondateur de son existence? Jusqu'à présent, la psychanalyse a plus parlé des fantasmes que de la réalité, mais il faut attirer l'attention sur le fait que certaines réalités risquent de dépasser la capacité de fantasmer d'un enfant, qui deviendra un adulte, et de dépasser aussi nos capacités de thérapeute.

Nous risquons de créer une génération d'enfants élevés dans des ruptures de continuité d'existence dues à la résidence alternée, dans une indifférenciation entre un parent et un tiers étranger, dans une indifférenciation concernant les sexes, et dans une absence de repères généalogiques. En tant que psychanalyste qui ne raisonne pas à partir de principes abstraits, mais qui voit les effets de ces changements sur certains enfants qui y sont confrontés, j'affirme que nous jouons aux apprentis sorciers concernant le devenir de notre société.

\section{Conclusion}

La structure œdipienne est toujours présente dans le développement psychique de l'être humain, mais pour se déployer, elle a besoin, d'un environnement pareexcitant et porteur de limites cohérentes dans les registres pulsionnels et narcissiques. Face aux modifications du fonctionnement groupal, cette structure n'est souvent 
plus assez forte pour organiser correctement le fonctionnement psychique de l'individu et diverses sortes de «dérives» peuvent apparaître. Les sentiments œdipiens sont douloureux en soi; oui, nos parents nous ont fabriqués en étant ensemble dans un lit; oui, comme enfant, nous sommes exclus partiellement du monde libidinal des adultes. La confrontation à une telle réalité peut, au prix d'une conflictualisation intra-psychique, permettre l'apparition de fantasmes originaires par lesquels l'enfant parvient cependant à se situer comme sujet actif. Mais les «nouveaux» modes de vie favorisent l'évitement du conflit œedipien. Il serait trop long de développer ici une description des différentes manières dont la conflictualité œedipienne et son élaboration sont entravées en fonction des différents contextes. Ceci a été évoqué à propos de la résidence alternée. Dans les couples recomposés, les mouvements de jalousie œdipienne sont compliqués par l'hypersexualisation du parent dans l'esprit de l'enfant qui prend une dimension "perverse» car non liée à la tendresse parentale, et par le fait que l'enfant peut penser que son père ou sa mère est abandonné par l'autre parent et l'abandonne. Par ailleurs, tous les dispositifs d'union ou de conception qui sont énigmatiques pour l'enfant parce qu'ils dissocient la sexualité des adultes de la procréation risquent d'aboutir à une hyper sexualisation de sa pensée.

Le principe du fonctionnement psychique est d'éviter un conflit chaque fois que c'est possible. Et précisément, les mouvements d'indifférenciation sociétaux éloignent de l'investissement de la conflictualité intrapsychique et tendent vers un appauvrissement de l'imaginaire dans le sens d'une réduction à un niveau plus primitif et d'une « déliaison» visant à tout réduire à un sexuel brut. Ceci me paraît grave et difficilement réversible.

Maurice Berger

Chef de service en pédopsychiatrie au CHU de Saint Étienne (France), psychanalyste. Pavillon 20 - Hôpital Bellevue - 25 bd Pasteur CHU - 42055 SAINT ETIENNE CEDEX 2 - FRANCE maurice.berger@chu-st-etienne.fr

\section{Notes}

1. Freud nomme «liaison» une opération qui tend à limiter le libre écoulement des excitations, à relier les représentations en elles, à constituer et à maintenir des formes relativement stables, donc in fine à organiser le monde pulsionnel de l'enfant.

2. Les prénoms des enfants cités dans cet article ont tous été changés

3. Seule une acceptation collective de certaines limites permet d'éviter qu'une réponse éducative ou thérapeutique contenante ne soit vécu instantanément comme sadique.

\section{Références}

AULAGNIER, P., 1984, L'apprenti historien et le maître-sorcier, PUF, Paris.

BERGER, M., 1986, Entretiens familiaux et champ transitionnel, Paris, PUF, repris in M. Berger, 1995, Le travail thérapeutique avec la famille, Dunod, Paris.

BERGER, M, 2008, Voulons-nous des enfants barbares?, Dunod, Paris.

BION, W. R., 1959/1965, Recherches sur les petits groupes, PUF, Paris. 
DONNET, J. L., 1993, Le Surmoi freudien et son ambigüité, Bulletin $n^{o} 1$ du groupe méditerranéen de la Société psychanalytique de Paris, 21-33.

FREUD, S., 1914/1973, Pour introduire le narcissisme, in La vie sexuelle, PUF, Paris.

FREUD, S., 1915/1985, Considérations actuelles sur la guerre et sur la mort, Payot, Paris.

FREUD, S., 1920/1985, Au-delà du principe de plaisir, in Essais de psychanalyse, Payot, Paris.

GUIGNARD, F., 2009, Le psychanalyste et l'enfant dans la société occidentale d'aujourd'hui, conférence faite à la Société belge de psychanalyse.

IZARD, E., 2009, Troubles psychiques observés chez les enfants vivant en résidence alternée non conflictuelle, in Neuropsychiatrie de l'enfant et de l'adolescent (à paraître).

LACROIX, M. B., 1999, Nous sommes tous des volés voleurs, in Enfants terribles, enfants féroces, Erès, Ramonville, 159-160.

LEVASSEUR, G., 2009, Ados: la fin de l'innocence. Enquête sur une sexualité à la dérive, Max Milo, Paris.

LEVY SOUSSAN, P., 2008, Grossesse pour autrui : risques pour soi et pour autrui, exposé fait à la Commission des lois du Sénat le 15 avril. 